\title{
Design of Fourth Order Digital PLLs Using Filter Prototypes
}

\author{
Brian Daniels, Ronan Farrell \\ Institute of Microelectronics and Wireless Systems, \\ National University of Ireland Maynooth, \\ County Kildare, Ireland
}

\begin{abstract}
In this paper an investigation of different filter prototypes and their applicability to digital phase locked loop design is carried out. A novel design technique using the superior filter prototype for the $4^{\text {th }}$ order Digital PLL is also introduced. The optimum choice of each design parameter is considered, while maintaining realisable component values as a priority. Finally the proposed design technique is used to design a $4^{\text {th }}$ order Digital PLL with optimum filter cut-off, stability and lock time. This $4^{\text {th }}$ order design method is an improvement on existing methods that exist in the literature to date, this is verified using simulation of a Digital PLL designed using the proposed technique.
\end{abstract}

\section{INTRODUCTION}

The Digital PLL (DPLL) is a versatile component block widely used in electronics for operations such as frequency synthesis, clock data recovery, and demodulation. The DPLL system consists of a phase frequency detector (PFD), a charge pump $(\mathrm{CP})$, and a voltage controlled oscillator (VCO), all of which are vital to the operation of the DPLL. The DPLL may also include a low pass loop filter (LF) or a frequency divider. A typical DPLL is shown in Fig. 1. The first order DPLL, with no loop filter is globally stable but produces large frequency jitter (phase noise) on the output signal that is intolerable for most applications. The solution is to include a simple RC low pass filter at the output of the $\mathrm{CP}$ to reduce this jitter. However discrete $V_{C}$ voltage jumps still exist due to voltage jumps across the filter resistor, these are commonly attenuated by including an additional ripple capacitor $\left(\mathrm{C}_{2}\right.$ in Fig. 2$)$ in parallel with the loop filter, increasing the PLL order to three, Fig. 2.

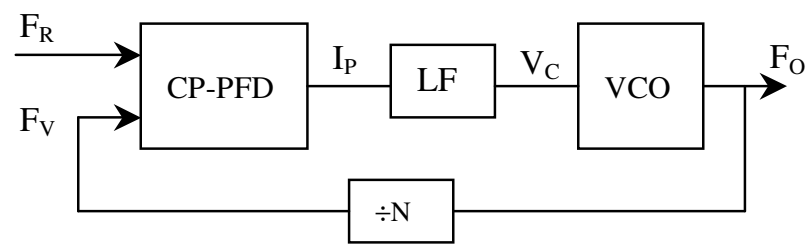

Fig. 1. DPLL Loop Block Diagram

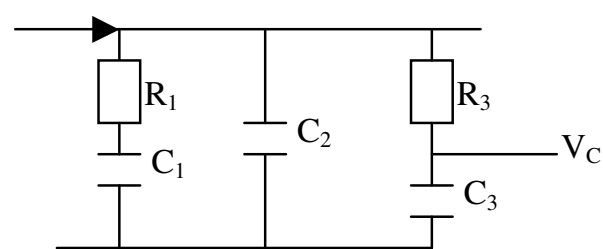

Fig. 2. $4^{\text {th }}$ order DPLL loop filter structure

The loop filter performs two operations on the $\mathrm{CP}$ output; first it converts the discrete like current output of the $\mathrm{CP}$ to a continuous DC like voltage for operation by the VCO; and second it attenuates high-frequency noise on the control voltage signal. It is necessary to eliminate noise on the control voltage, as this will be represented as jitter on the PLL output signal. Higher order filters provide greater attenuation of this jitter. For the purpose of low noise operation, passive filters are preferred to active filters, however this adds some restriction on the choice of filter transfer function.

The DPLL is a highly non-linear system and is further complicated by the fact that the variable of interest around the loop changes from phase to voltage, in the PFD, CP, and LF, and back to phase in the VCO. The DPLL can be approximated to a linear transfer function by replacing the $\mathrm{CP}$ PFD block in Fig. 1 with a multiplier and gain component $\left(\mathrm{K}_{\mathrm{P}}\right)$, and replacing the VCO block with an integrator and a gain component $\left(\mathrm{K}_{\mathrm{V}}\right)$, as in [1]. The closed loop transfer function, $\mathrm{H}_{\mathrm{CL}}(\mathrm{s})$, is expressed as in (1).

$$
H_{C L}(s)=\frac{N K_{V} I_{P} F(s)}{2 \pi N s+K_{V} I_{P} F(s)}
$$

The $4^{\text {th }}$ order DPLL loop filter F(s), has a structure as in Fig. 2, and is expressed as in (2) below. The passive filter structure of Fig. 2 is designed by choosing a $\omega_{c}$, of approximately $1 / 10^{\text {th }}$ the reference frequency, $\omega_{R}$, this is a rule of thumb based on recommendations and empirical results of [1]. Knowing $\omega_{c}$ the components $R_{1}$ and $C_{1}$ are chosen from the solution to $\omega_{c}=$ $1 / R_{1} C_{1}$, and $C_{2}$ is chosen to be $1 / 10^{\text {th }}$ of $C_{1}$. The VCO and CP gains are then chosen using rule of thumb or a design criterion such as [1] to assure the system stability.

$$
F(s)=\frac{C_{1} R_{1} s+1}{C_{1} C_{2} C_{3} R_{1} R_{3} s^{3}+\left(C_{1} C_{2} R_{1}+C_{1} C_{3} R_{3}+C_{2} C_{3} R_{3}+C_{1} C_{3} R_{1}\right) s^{2}+\left(C_{1}+C_{2}+C_{3}\right) s}
$$


Once the low order filter components are chosen, high order elements are added to attenuate out-of-band noise. This paper uses the piecewise linear method (PWL) of [2], along with common filter prototypes to design stable $4^{\text {th }}$ order DPLLs with optimum stability, lock time, and $\omega_{\mathrm{c}}$ characteristics. In section II filter prototypes, as an alternative to traditional loop filters, and their adaptability to the DPLL are discussed. In section III a novel design technique is proposed. The proposed technique uses filter prototypes to place filter poles in-band to give optimum noise attenuation. In section IV the optimum filter prototype are considered, and an example of a design is given. Finally in section $\mathrm{V}$ conclusions are presented.

\section{FILTER PRototyPe AND SySTEM EQUATIONS}

In [3] the Bessel filter prototype has been used to design the $3^{\text {rd }}$ order DPLL. Bessel prototypes are specifically chosen due to its linear phase offset in the filter pass-band. This however is an unnecessary requirement as the DPLL reference frequency is constant, and any phase offset is corrected during the operation of the DPLL. This is verified by comparing the phase offset of the traditional DPLL to filter prototype phase offsets in the pass-band, as in Fig. 3 for a cut-off frequency of $10 \mathrm{MHz}$. It is clear that the traditional DPLL has a phase offset that is much more non-linear in the pass-band then the selected prototypes. For this reason the design method need not be restricted to the Bessel prototype but may also include frequency selective prototypes such as Butterworth and Chebyshev. The application of each of these filter prototypes is considered and compared in this section.

The DPLL filter prototype design method determines component values of the filter by equating the prototype transfer function denominator with the denominator of $\mathrm{H}_{\mathrm{CL}}(\mathrm{s})$ in (1). $H_{C L}(s)$ has one zero located at $s=-1 / R_{1} C_{1}$ and in the case of a forth order DPLL it has four poles. Because only the prototype and $\mathrm{H}_{\mathrm{CL}}(\mathrm{s})$ denominators are equated, and not the numerators, the filter prototype is required to be an all pole system with no zeros; the prototypes that match this criterion are the Bessel, Butterworth and Chebyshev type 1 prototypes.

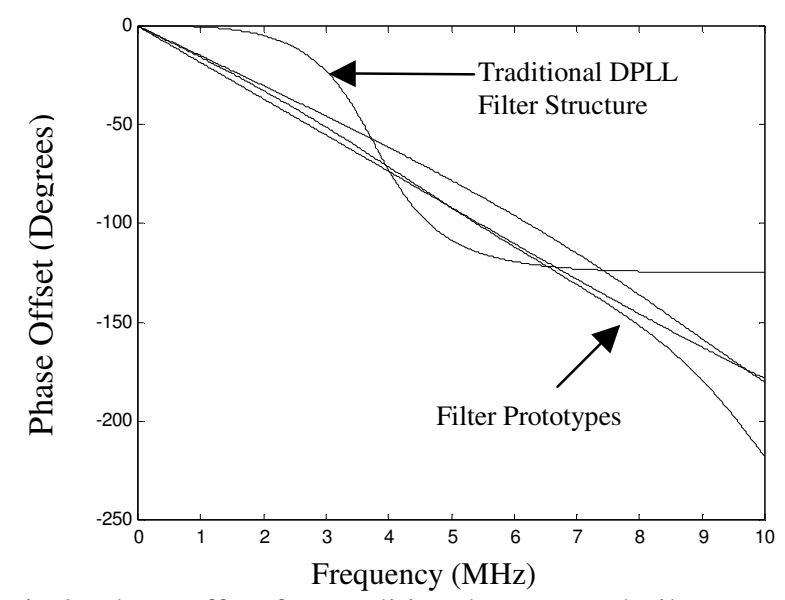

Fig 3: Phase offset for Traditional DPLL and Filter Prototypes
TABLE 1:

\begin{tabular}{|c|c|c|c|c|c|}
\multicolumn{8}{|c}{ NORMALISED FILTER PROTOTYPE COEFFICIENTS } \\
\hline Prototype & Bessel & Butter & $\begin{array}{l}\text { Cheby } \\
\mathrm{R}=0.1\end{array}$ & $\begin{array}{c}\text { Cheby } \\
\mathrm{R}=0.5\end{array}$ & $\begin{array}{c}\text { Cheby } \\
\mathrm{R}=0.969\end{array}$ \\
\hline$\alpha$ & 3.124 & 2.6131 & 1.804 & 1.197 & 0.9637 \\
\hline$\beta$ & 4.392 & 3.4142 & 2.627 & 1.717 & 1.464 \\
\hline$\delta$ & 3.201 & 2.6131 & 2.026 & 1.025 & 0.7541 \\
\hline$\varepsilon$ & 1 & 1 & 0.8285 & 0.3791 & 0.2795 \\
\hline$\chi$ & 1 & 1 & 0.819 & 0.3578 & 0.25 \\
\hline
\end{tabular}

The transfer function of these prototypes is given in (3), where $\alpha, \beta, \delta$, and $\varepsilon$ are the normalised coefficients from Table 1 above.

$$
H_{B E S S E L}(s)=\frac{\chi \omega_{C}^{4}}{s^{4}+\alpha \omega_{C} s^{3}+\beta \omega_{C}^{2} s^{2}+\delta \omega_{C}^{3} s+\varepsilon \omega_{C}^{4}}
$$

The component values of the DPLL are calculated using (4-8). These component values produce optimum cut-off characteristics for the DPLL filter structure.

$$
\begin{aligned}
& C_{1}=\frac{\beta \delta K\left(M_{1} M_{2}+1\right)-\alpha \varepsilon M_{1} M_{2} K}{N \delta \varepsilon \omega_{C}^{2}\left(M_{1} M_{2}+\frac{1}{M_{1}}\right)} \\
& C_{2}=\frac{\frac{\alpha M_{1} M_{2} K}{N \delta \omega_{C}^{2}}-C_{1} M_{2}-C_{1}}{M_{1} M_{2}+1} \\
& R_{1}=\frac{\delta}{\varepsilon C_{1} \omega_{C}} \\
& R_{3}=\frac{R_{1}}{M_{2}} \\
& C_{3}=\frac{C_{1}}{M_{1}}
\end{aligned}
$$

To solve (4-8) there are four unknown parameters that need to be considered, $\mathrm{K}, \mathrm{M}_{1}, \mathrm{M}_{2}$, and $\omega_{\mathrm{c}}$. Each of these will be considered in the next section.

\section{Proposed Design TECHNIQUE}

In this section the PWL method of [2] is used to determine the optimum choice of $\mathrm{K}, \mathrm{M}_{1}, \mathrm{M}_{2}$ and $\omega_{\mathrm{c}}$, from the previous section, where $\mathrm{K}$ is equal to $\mathrm{K}_{\mathrm{P}} \mathrm{K}_{\mathrm{V}}$. Using the above techniques and optimised parameters, an optimum DPLL system is designed, simulated, and shown to be an improvement over existing techniques.

The forth order DPLL has six unknown component values yet the filter prototype provides only four equations, the solution to this is to introduce two ratios $M_{1}$ and $M_{2}$ (7-8). These parameters define the location of the filter pole $\mathrm{P}_{4}$ in Fig. 4 . Ideally we require that the $\mathrm{H}_{\mathrm{CL}}(\mathrm{s})$ poles be located at the same point as the prototype poles, however this is not feasible for the passive loop filter structure of Fig. 2. The poles are placed as close to the ideal location as possible. 


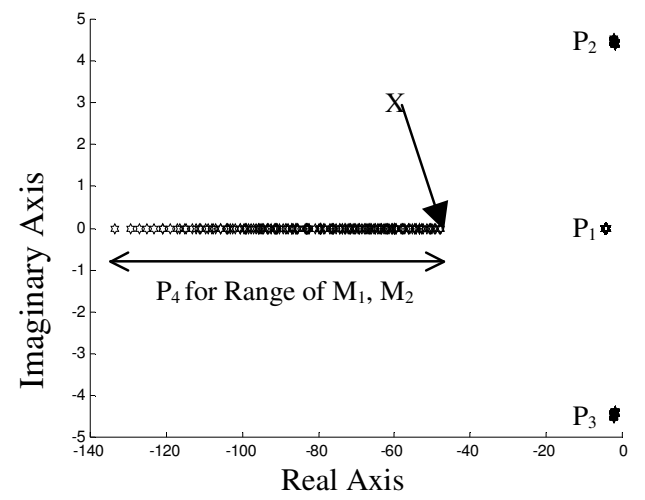

Fig. 4. Pole Locations for range of $\mathrm{M}_{1}$ and $\mathrm{M}_{2}$

Fig. 4 shows that increasing $\mathrm{M}_{1}$ and $\mathrm{M}_{2}$ causes pole $\mathrm{P}_{4}$ to move and $\mathrm{P}_{1}, \mathrm{P}_{2}$ and $\mathrm{P}_{3}$ to remain constant. Similarly Fig. 5 shows that varying $\mathrm{M}_{1}$ and $\mathrm{M}_{2}$ affects the roll-off of the system magnitude. Ideally we require a value of $\mathrm{M}_{1}$ and $\mathrm{M}_{2}$ that will produce the sharpest roll-off and therefore best filter cut-off characteristics. This occurs at the point where $\mathrm{P}_{4}$ is closest to $P_{1}$. The trajectory of $P_{4}$ as $M_{1}$ and $M_{2}$ change is irregular, it initially moves closer to $P_{1}$ for increasing $M_{1}$ and $M_{2}$. When $P_{4}$ reaches the point $X$, in Fig. 4 , the pole turns and moves away from $P_{1}$. The optimum choice of $M_{1}$ and $M_{2}$ is the point where $\mathrm{P}_{4}$ lies at $\mathrm{X}$. The optimum location is determined from the denominator of $\mathrm{H}_{\mathrm{CL}}(\mathrm{s})$. If the denominator of $\mathrm{H}_{\mathrm{CL}}(\mathrm{s})$ is $\mathrm{D}(\mathrm{s})$, as in (9), then the minimum value of $\mathrm{P}_{4}$ occurs at a minimum value of $A$, where $A$ is shown in (10) below.

$$
D(s)=s^{4}+A s^{3}+B s^{2}+C s+D
$$

The minimum point can be determined by solving $\mathrm{A}$ for all $\mathrm{M}_{1}$ and $\mathrm{M}_{2}$. Equations (4-8) are dependent on the choice of gain $\mathrm{K}$. If we solve $\mathrm{H}_{\mathrm{CL}}$ (s) in (1) using (4-8) we find that

$$
H_{C L}(s)=\frac{\text { NKnum }}{\text { NKden }+ \text { Knum }}
$$

The $\mathrm{K}$ 's in (11) all cancel, so $\mathrm{H}_{\mathrm{CL}}(\mathrm{s})$ is independent of $\mathrm{K}$, any change in $\mathrm{K}$ is reflected by a proportionate change in the component values.

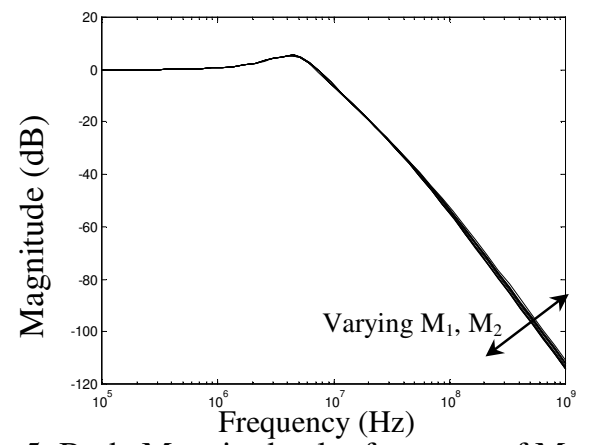

Fig. 5. Bode Magnitude plot for range of $M_{1}$ and $M_{2}$
This has the effect of keeping the system poles in their optimum location making $\mathrm{K}$ insignificant with respect to the system response. However $\mathrm{K}$ has an effect on the filter component values (4-8) and is chosen solely to achieve realisable values.

The choice of $\omega_{C}$ is crucial for a stable DPLL system design. Traditionally it was suggested to choose $\omega_{C}$ to be at least $1 / 10^{\text {th }}$ of $\omega_{R}$, otherwise the CTA would become invalid and the loop filter would pass large amounts of in-band noise causing instability in the DPLL. The traditional choice of $\omega_{C}$, from $[3,4,5]$ is plotted against the normalised system lock time $\left(t_{\mathrm{LCK}} \mathrm{F}_{\mathrm{R}}\right)$, as dots in Fig. 6 , and has a minimum $\omega_{\mathrm{C}} / \omega_{\mathrm{R}}$ ratio of $1 / 13^{\text {th }}$. Ideally we require a $\omega_{\mathrm{C}}$ close to $\omega_{\mathrm{R}}$, to reduce out-ofband noise, but the CTA must still be valid. Using the PWL method we can determine the DPLL lock time as $\omega_{\mathrm{C}}$ approaches $\omega_{\mathrm{R}}$. This is shown in Fig. 6 as a line for a range of $\omega_{\mathrm{C}} / \omega_{\mathrm{F}}$. In Fig. 6 the CTA begins to break down at $\omega_{\mathrm{C}} / \omega_{\mathrm{R}}$ greater then 0.1 . The system lock time is also reduced as $\omega_{C}$ approaches $\omega_{\mathrm{R}}$. Also we know that the out-of-band noise attenuation will be greater the closer $\omega_{C}$ is to $\omega_{R}$. Therefore we require $\omega_{C}$ to be as close to $\omega_{\mathrm{R}}$ as possible, but avoiding the CTA break down point. Identification of the breakdown point can improve the design process. This is achieved using the PWL numerical solution method of [2].

Using (4-8), choosing the optimum $\omega_{\mathrm{C}}$ close to $\omega_{\mathrm{R}}$ for minimum lock time, and finally choosing optimum $\mathrm{M}_{1}$ and $\mathrm{M}_{2}$ for the sharpest roll-off, an optimum, stable, and realizable $4^{\text {th }}$ order DPLL is designed. This is demonstrated in the next section.

\section{OPTIMUM FILTER PROTOTYPE AND DESIGN EXAMPLE}

In this section each filter prototype from Table 1 is considered. The best filter prototype is then applied to the design method of the previous section. In Fig. 7 the lock time (dashed line) and steady state error (continuous line) for each prototype is shown.

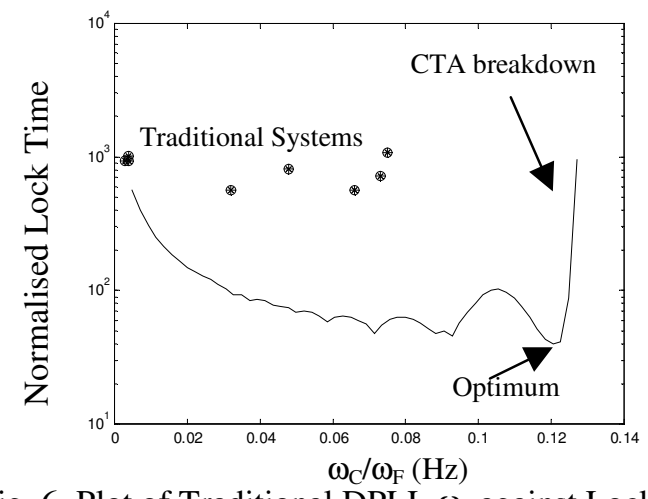

Fig. 6. Plot of Traditional DPLL $\omega_{\mathrm{C}}$ against Lock Time

$$
A=\frac{\omega_{C}\left(M_{1} M_{2}+1\right)}{\delta}\left[\frac{\left(M_{2}+1\right)\left(\beta \delta\left(M_{1} M_{2}+1\right)-\alpha \varepsilon M_{1} M_{2}\right)}{\alpha \varepsilon\left(M_{1}^{2} M_{2}^{2}+M_{2}\right)-\left(M_{2}+1\right)\left(\beta \delta\left(M_{1} M_{2}+1\right)-\alpha \varepsilon M_{1} M_{2}\right)}\right]
$$


It is clear from both plots that the Chebyshev filter returns the best results for both lock time and steady state error for all Chebyshev ripple parameters, $\mathrm{R}$. As discussed earlier the value of $\mathrm{K}$ is insignificant in terms of the system response and is chosen here using traditional rule-of-thumb, the values are $\mathrm{K}_{\mathrm{V}}$ $=62.8 \mathrm{MHz} / \mathrm{V}$ and $\mathrm{I}_{\mathrm{P}}=10 \mu \mathrm{A}$. The filter ratios $\mathrm{M}_{1}$ and $\mathrm{M}_{2}$ are chosen to produce the lowest possible lock time and optimum location of $\mathrm{P}_{4}$. The value of $\mathrm{R}$ allows the designer to trade-off between faster lock time and better steady state error. As $\mathrm{R}$ is increased $\omega_{C}$ approaches $\omega_{R}$, this is illustrated in Fig. 8. The lock time and steady state error can be varied by optimally choosing R. Fig. 9 shows the lock time (dashed line) and steady state error (continuous line) for a range of $\mathrm{R}$. The minimum lock time and steady state error is found to occur at a value of $\mathrm{R}$ equal to 0.707 .

Consider the design of a $20 \mathrm{MHz}$ DPLL system using the proposed design method. This system has a feedback divide ratio of 10 , and gains $\mathrm{K}_{\mathrm{V}}=300 \mathrm{MHz} / \mathrm{V}$ and $\mathrm{I}_{\mathrm{P}}=10 \mu \mathrm{A}$. A Chebyshev filter prototype with an $\mathrm{R}$ of 0.707 is used. The optimum choice of $\mathrm{M}_{1}$ and $\mathrm{M}_{2}$ are found using the PWL method to give optimum lock time and optimum location of $\mathrm{P}_{4}$. For this particular system $\mathrm{M}_{1}$ is chosen to be 12 , and $\mathrm{M}_{2}$ is 1.4 . From these chosen parameter values the filter components are calculated to be $\mathrm{C}_{1}=12.4 \mathrm{pF}, \mathrm{C}_{2}=1.98 \mathrm{pF}, \mathrm{C}_{3}=1.04 \mathrm{pF}$, $\mathrm{R}_{1}=17.3 \mathrm{k} \Omega$ and $\mathrm{R}_{2}=12.4 \mathrm{k} \Omega$. The response of this system is demonstrated in Fig. 10.

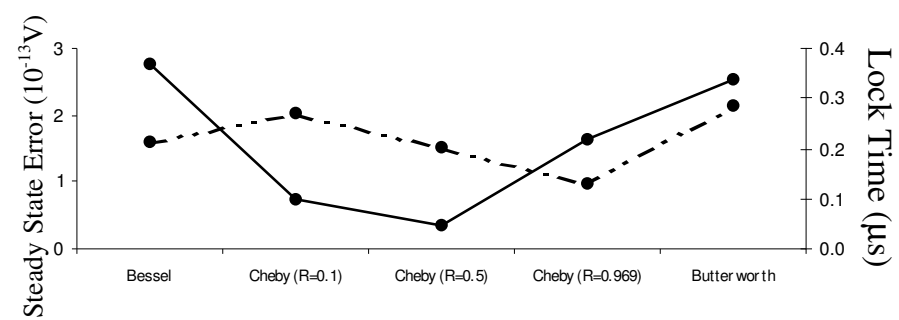

Fig. 7. Lock Time and steady state error for Filter Prototypes
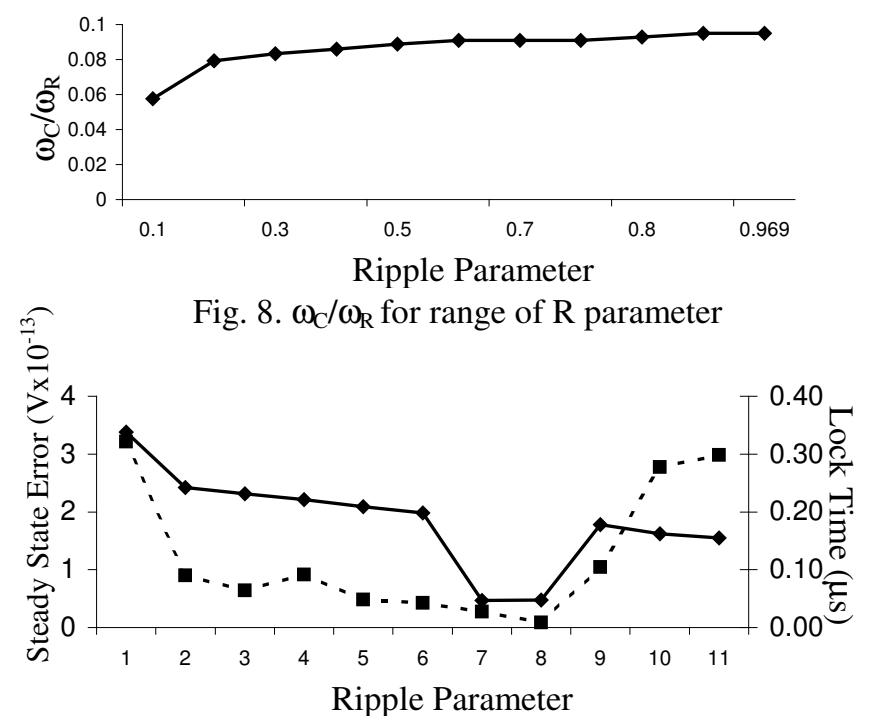

Fig. 9. Lock Time (dashed line) and steady state error (continuous) for Chebyshev filters

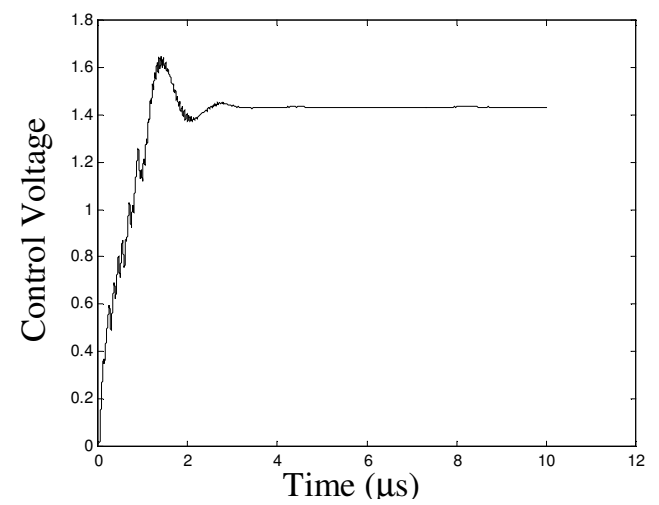

Fig. 10. System Response of a $20 \mathrm{MHz} 4^{\text {th }}$ order DPLL

\section{CONCLUSION}

In this paper filter prototypes are used to design a $4^{\text {th }}$ order DPLL. The system components are found by the optimum choice of $\omega_{C}$ and filter ratios $M_{1}$ and $M_{2}$. The optimum values for steady state error, lock time and sharp filter roll-off are found through the numerical of (10) and the PWL model of [2]. The Chebyshev, Bessel, and Butterworth filter prototypes are each considered and the best filter prototype is found to be the Chebyshev with a ripple value of 0.707 . Using this Chebyshev filter and the optimum $\omega_{C}, M_{1}$ and $M_{2}$, a stable, fast locking, low noise $4^{\text {th }}$ order DPLL is demonstrated.

\section{ACKNOWLEDGEMENTS}

The authors acknowledge the support of the Enterprise Ireland Commercialization fund.

\section{REFERENCES}

[1] F.M. Gardner, "Charge pump phase-lock loops," IEEE Trans. Commun., vol. COM-28, Nov 1980, 1849-1858.

[2] B Daniels, R Farrell, "Stability analysis of high frequency digital phase locked loops using piecewise linear model," Proceedings of Irish signals and systems conf, Dublin 2006

[3] S Mirabbasi, and K Martin, "Design of loop filter in phase-locked loops," IEEE Electronic Letters 1999, Vol. 35, Issue. 21, pp. 1801-1802

[4] R Best, "Phase-locked loops, design, simulation, and applications," $3^{\text {rd }}$ edition (McGrath-Hill 1997).

[5] D Banerjee, "PLL performance, simulation, and design," (Dean Banerjee Pubns 2003).

[6] B Daniels, R Farrell, G Baldwin, "Modeling and design of highorder phase locked loops," proceedings of SPIE, volume 5837, Seville 2005 